Ergod. Th. \& Dynam. Sys. (1989), 9, 609-618

Printed in Great Britain

\title{
Rokhlin's School in ergodic theory
}

\author{
SERGEY YUZVINSKY \\ University of Oregon, Eugene, Oregon 97403, USA
}

(Received 20 April 1986 and revised 25 September 1987)

\section{Introduction}

Ergodic theory is one of several fields of mathematics where the name Vladimir Abramovich Rokhlin (spelled also 'Rochlin' and 'Rohlin') is well known to the specialists. That name is attached to some very often used theorems, but the goal of this paper is not just to remind the reader of these theorems. I put them in the context of the general development of ergodic theory during the thirty years 1940-70. Most of all, I want to emphasize that Rokhlin was not only a researcher producing powerful results but also a founder of schools at the two best Universities in the USSR. For at least 10 years (1958-68) these schools dominated ergodic theory. This paper is not biographical. Rokhlin's life certainly deserves a better biographer. However, I mention certain circumstances of a non-mathematical nature where it seems to be appropriate.

This paper is divided into three sections corresponding to three easily distinguishable periods in Rokhlin's work in ergodic theory. The first section called BE (before entropy) corresponds to the first period which lasted from 1940 to 1949 . It was devoted to axiomatization, spectral invariants, and mixing properties. The following 9 years were a quiet period in ergodic theory. In particular, Rokhlin was working in topology and apparently did not plan to return to ergodic theory. In 1958, A. Kolmogorov introduced a new invariant: the entropy of a measure preserving transformation. This started a new era in ergodic theory that I will call ET (entropy theory) and attracted Rokhlin back to the area. The second section of this paper is devoted to the results of the seminar organized by Rokhlin at the Moscow State University and covers approximately 1958-1959. We also follow the later history of several important problems formulated in this seminar. Finally, the third section is devoted to Rokhlin's seminar at the Leningrad State University in 1960-1970.

D. Ornstein's paper [22] on the isomorphism of Bernoulli automorphisms with equal entropy started yet another period in ergodic theory. This period can be characterized by the development of methods of more combinatorial nature that were harder to formalize. Many old problems were solved by these methods. At this point the center of pure ergodic theory or rather Bernoulli theory moved to the West. Perhaps this was one of the reasons why Rokhlin stopped doing active research in the area. Besides, he was busy proving his famous results in 4-dimensional topology. 


\section{1. $B E$}

Rokhlin obtained his first results in ergodic theory in 1940 when he was a student at Moscow University. They were published in short from only seven years later in $[\mathbf{2 5}, \mathbf{2 7}]$. Yet later the results of [25] appeared in detail in [28]. During these seven years the author of the results fought the German Army with the Moscow University Volunteer Brigade, was captured, survived, finished the war with the Red Army in Berlin, was jailed by his compatriots as a survivor of a POW camp, and eventually released, having surprisingly preserved his ability to fruitfully do mathematics.

In [28], Rokhlin solved some problems suggested to him by Kolmogorov. First of all, he characterized the measure spaces isomorphic (measure-theoretically) to an interval with the Lebesgue measure augmented by a sequence of points of positive measure. The notion of such a space (called Lebesgue space) was to become a fundamental concept of ergodic theory. According to [28], a space $X$ with a measure $\mu(\mu(X)=1)$ is a Lebesgue space if and only if there exists a countable basis $\left(A_{n}\right)_{n=0}^{\infty}$ of the $\sigma$-algebra of measurable sets of $X$ which separates points and such that $X$ is complete with respect to it. The latter condition means that $\bigcap_{n=0}^{\infty} B_{n} \neq \varnothing$ for every choice $B_{n}=A_{n}$ or $B_{n}=X \backslash A_{n}$. A similar characterization of Lebesgue spaces was given by $P$. Halmos and J. von Neumann in [10].

Secondly, Rokhlin developed the theory of measurable partitions. He defined a measurable partition of $X$ as a partition generated by a countable system of measurable sets. Equivalently, it is the partition into preimages of points under a measure preserving mapping of $(X, \mu)$ onto another Lebesgue space. The main property of a measurable partition $\xi$ is that it affords a canonical system of measures on its elements. This means that each element $C$ of $\xi$ can be provided with a Lebesgue measure $\mu_{C}$ such that for every (measurable) set $A \subset X$

$$
\mu(A)=\int_{X / \xi} \mu_{C}(A \cap C) d \mu_{\xi}
$$

where $\mu_{\xi}$ is the measure induced by $\mu$ on the factor space $X / \xi$ (of all elements $C)$. It turns out that the measurable partitions allow a complete classification. In the most important case where $\mu$ is continuous every (measurable) partition with continuous measures on its elements is isomorphic to the partition of the unit square into vertical intervals.

Rokhlin observed that the set of all measurable partitions provided with the natural order $(\xi<\eta$ if $\eta$ is a refinement of $\xi$ ) is a complete lattice. Of course here as virtually everywhere else in measure theory the folowing convention is applied: the objects which are different only on a set of measure 0 are identified. The importance of that observation would become completely clear only in entropy theory.

In the same year when his first results were published, Rokhlin published the papers $[29,30]$. There, he summed up his activity in ergodic theory, not planning to work in this area any more. The paper [29] is an exposition of the theory of measure-preserving transformations overlapping with similar expositions by E. Hopf 
[14] and P. Halmos [13]. The precise and exhaustive style of the paper became typical of all other expositions by Rokhlin.

Among Rokhlin's own results from this paper there are two that influenced the further development of ergodic theory most significantly. The first one is the theorem about the strong approximation of an aperiodic automorphism by periodic automorphisms. More precisely, we let $d$ be the metric on the group of all automorphisms of a Lebesgue space $X$ defined by $d(T, S)=\mu(x \in X \mid T x \neq S x)$. Halmos proved in [12] that for every aperiodic automorphism $T$ and every natural number $p$ there exists an automorphism $S$ of period $p$ such that $d(T, S)<4 / p$. Rokhlin showed that instead of $4 / p$ one can put $1 / p+\varepsilon$ for every positive $\varepsilon$. This result became fundamental in the approximation theory developed by A. Katok and A. Stepin [18].

Another important result of the paper is the theorem about mixing automorphisms. Let us recall that an automorphism $T$ is said to be mixing if for every pair $(A, B)$ of measurable sets $\lim _{n \rightarrow \infty} \mu\left(T^{n} A \cap B\right)=\mu A \cdot \mu B$. Every mixing automorphism has a continuous spectrum. Rokhlin's theorem states that the mixing automorphisms form a set of first category in the set of all automorphisms provided with metric $d$. Earlier, Halmos [12] proved that the set of automorphisms with continuous spectrum is an everywhere dense $G_{\delta}$. Hence, roughly speaking, a typical automorphism has a continuous spectrum but is not mixing.

Since the beginning of his work in ergodic theory Rokhlin was interested in a special class of transformations: the endomorphisms of compact commutative groups. Since a compact group $G$ with a countable basis of open sets provided with Haar measure $\mu$ is a Lebesgue space and any epimorphism $T$ of $G$ preserves $\mu, T$ can be studied from the point of view of ergodic theory. Conditions of ergodicity of such an endomorphism $T$ on a commutative group had already been obtained by Rokhlin in 1941. They were also published by Halmos in [11]. In [30], Rokhlin published his proof of these conditions. He also defined a new invariant of an endomorphism: the mixing of order $r$ for every positive integer $r$. If $r=1$ this is the usual mixing. As an example he proved that an endomorphism of a compact commutative group is the mixing of all orders.

Rokhlin hoped that the mixing of order greater than one was not a spectral invariant. This question which stimulated a lot of important work in ergodic theory is still open. It is not even known if the usual mixing implies the mixing of higher orders. The most recent result in this direction was obtained by S. Kalikow. A weaker version of it was published in [63]. Also a similar notion of "weakly mixing of order $r$ ' was studied by H. Furstenberg in [62].

\section{ET, Moscow seminar}

In 1958, Kolmogorov $[19,20]$ introduced a new invariant of an automorphism $T$ of a Lebesgue space: the entropy $h(T)$ of $T$. Kolmogorov's definition (or rather Sinai's modification in [42]) amounts to the following. For a partition $\xi$ of the space into sets $A_{1}, A_{2}, \ldots$ of positive measure the entropy $H(\xi)$ is defined by

$$
H(\xi)=-\sum_{i} \mu\left(A_{i}\right) \log \mu\left(A_{i}\right)
$$


Now, for an automorphism $T$ let

$$
h(T, \xi)=\lim _{n \rightarrow \infty} H\left(\bigvee_{i=0}^{n-1} T^{i} \xi\right) / n
$$

and

$$
h(T)=\sup _{\xi} h(T, \xi)
$$

where the supremum is taken with respect to all finite partitions. Kolmogorov proved that if a finite entropy partition $\xi$ is a generator of $T$, i.e. $\bigvee_{-\infty}^{\infty} T^{n} \xi=\varepsilon$ (the partition into points), then $h(T, \xi)=h_{1}(T)$ is independent of $\xi$ (see also [42]). The identity $h(T)=h_{1}(T)$ was proved by Rokhlin [36]. This immediately gives examples of non-isomorphic Bernoulli schemes. The Kolmogorov-Sinai definition can be easily generalized to endomorphisms.

Kolmogorov's discovery started a new era in ergodic theory and attracted Rokhlin back to this field. From October 1958 to May 1959 Rokhlin ran an ergodic theory seminar in the Department of Mechanics and Mathematics of the Moscow University (without being employed by the University). Many young talented mathematicians such as L. Abramov, I. Girsanov, B. Gurevich, L. Meshalkin, M. Pinsker, and Ya. Sinai took part in the seminar. The results proved by Rokhlin himself and by other participants of the seminar are presented in Rokhlin's expository paper [32].

First of all, several basic properties of entropy were proved. Among them are

(1) $h\left(T^{n}\right)=|n| h(T)$ for an arbitrary integer $n[42]$;

(2) $h(S \times T)=h(T)+h(S)$ where $S \times T$ is the direct product of the automorphisms $S$ and $T[31]$

(3) $h(S) \leq h(T)$ for every factor automorphism $S$ of $T[31]$;

(4) $h\left(T_{A}\right)=h(T) / \mu(A)$ where $T_{A}$ is the automorphism induced by $T$ on the subspace $A$ such that $\mu\left(\bigcup_{n=-\infty}^{\infty} T^{n} A\right)=1[1]$.

Secondly, the problem of computing $h(T)$ for an automorphism $T$ without an explicitly given generator was considered. In [32], there are several examples of conditions on a sequence $\left(\xi_{n}\right)$ of partitions that guarantee the convergence $h\left(T, \xi_{n}\right) \rightarrow$ $h(T)$. For example [31], if $\left(\xi_{n}\right)$ is an increasing sequence and $\bigvee_{n} \xi_{n}=\varepsilon$ then $\lim h\left(T, \xi_{n}\right)=h(T)$. Using a similar condition, Sinai [42] computed the entropy of certain automorphisms of the torus, which started a series of interesting examples from topology and algebra.

Another natural question was to study relations between the entropy and the spectrum of an automorphism. In [31], Rokhlin proved that every automorphism with positive entropy has a Lebesgue component of infinite multiplicity in its spectrum. He also characterized the automorphisms with zero entropy without using the entropy and showed that they form a dense $G_{\delta}$ in the space of automorphisms.

Introducing his new invariant, Kolmogorov at the same time introduced a new class of automorphisms that he called 'quasi-regular'. Later Rokhlin started to call them Kolmogorov automorphisms. An automorphism $T$ is a Kolmogorov 
automorphism if there exists a partition $\zeta$ such that

$$
T \zeta>\zeta, \quad \bigvee_{n=-\infty}^{\infty} T^{n} \zeta=\varepsilon, \bigwedge_{n=-\infty}^{\infty} T^{n} \zeta=\nu
$$

$\nu$ being the trivial partition into one set. Pinsker in [24] proved that every Kolmogorov automorphism has completely positive entropy, i.e. $h(T, \xi)>0$ for every partition $\xi$. This condition turned out to be easier to work with than $(*)$. For instance, it is obvious that if an automorphism has completely positive entropy then its inverse and all its factor automorphisms have this property. At the same time, similar implications for the Kolmogorov automorphisms are quite non-trivial and are usually deduced from the Rokhlin-Sinai theorem [41] (see page 614). Pinsker also proved in [24] that an arbitrary automorphism $T$ has a unique maximal partition $\pi=\pi(T)$ such that $T \pi=\pi$ and the factor automorphism $T_{\pi}$ has zero entropy.

The factor endomorphism $S$ of a Kolmogorov automorphism $T$ with respect to a partition $\zeta$ satisfying (*) has the property $\wedge_{n=0}^{\infty} S^{-n} \varepsilon=\nu$. Rokhlin built a theory of these endomorphisms in [33], naming them exact endomorphisms. It turned out that interesting examples of exact endomorphisms can be found not only in probability theory but also in topological algebra (endomorphisms of tori) and in number theory. A beautiful example of the last type considered by Rokhlin is the Gauss endomorphism. It is given on the unit interval with the measure

$$
\mu(A)=\frac{1}{\ln 2} \int_{A} \frac{d x}{1+x}
$$

by the formula

$$
\Gamma x=\frac{1}{x}-\left[\frac{1}{x}\right]
$$

where $[a]$ is the integer part of a number $a$. It turned out that the endomorphism $\Gamma$ is exact and $h(\Gamma)=\pi^{2} / 6(\ln 2)^{2}$. This formula is closely related to continued fraction theory.

Finally, the participants of the seminar studied the notion of entropy for a (measurable) flow, i.e. a one-parameter group $\left\{S_{t}\right\}_{t \in \mathbf{R}}$ of automorphisms. Kolmogorov defined its entropy as

$$
h\left(\left\{S_{t}\right\}\right)=\sup _{t} \frac{h\left(S_{t}\right)}{t}, \quad t>0 .
$$

Abramov in [2] proved that $h\left(S_{t}\right)=|t| h\left(S_{1}\right), t \in R$, whence $h\left(\left\{S_{t}\right\}\right)=h\left(S_{1}\right)$. Kolmogorov also defined a new class of flows. A flow $\left\{S_{t}\right\}$ is called a Kolmogorov flow if there exists a partition $\xi$ such that $S_{t} \xi<S_{u} \xi$ for $t<u$,

$$
\bigvee_{-\infty<t<\infty} S_{t} \xi=\varepsilon \text { and } \bigwedge_{-\infty<t<\infty} S_{t} \xi=\nu
$$

Kolmogorov [19] and Sinai [44] proved that such a flow has Lebesgue spectrum of infinite multiplicity, Rokhlin proved that it is a mixing of all orders. Since the notion of a Kolmogorov flow came from probability theory it was an exciting problem to find examples of those flows among classical dynamical systems. Sinai [44] proved 
that the geodesic flow on compact manifolds of constant negative curvature is a Kolmogorov flow and computed the entropy of the flow.

Summing up in [32] the results of the seminar, Rokhlin stated several problems which determined the future development of the area. We will consider several of them.

\subsection{Isomorphism problem}

Is entropy a complete invariant on the class of automorphisms with completely positive entropy? On the class of Bernoulli automorphisms? Do these two classes coincide?

All these questions were solved by $D$. Ornstein, the second one positively [22], the third and hence the first one negatively [23]. That was a beginning of yet another new era in ergodic theory.

\subsection{Kolmogorov automorphisms}

Is any automorphism with completely positive entropy a Kolmogorov automorphism?

This question was solved positively in the paper [41] by Rokhlin and Sinai. They studied certain classes of invariant partitions satisfying more general conditions than (*). A partition $\zeta$ is said to be extremal with respect to an automorphism $T$ if

$$
T \zeta \geq \zeta, \quad \bigvee_{0}^{\infty} T^{n} \zeta=\varepsilon, \quad \bigwedge_{0}^{\infty} T^{-n} \zeta=\pi(T)
$$

The main result in [41] is the existence for every automorphism $T$ of an extremal partition $\zeta$ such that $h(T, \zeta)=h(T)$. If $T$ has completely positive entropy then $\pi(T)=\nu$ and $(* *)$ is equivalent to $T$ being a Kolmogorov automorphism.

\subsection{Generators}

Does every ergodic automorphism with finite entropy have a generator with finite entropy?

The problem was solved affirmatively by Rokhlin $[36,38]$. He introduced a metric on the set $Z$ of all partitions with finite entropy such that $Z$ is complete in this metric. The main theorem of [36] says that if $T$ is an aperiodic automorphism with finite entropy then the complement of the set of generators of $T$ in the set of partitions $\xi \in Z$ such that $h(T, \xi)=h(T)$ is of the first category. The existence of generators with finite entropy follows immediately. An easier theorem from [36] states that every aperiodic automorphism has a countable or finite generator. Similar results are proved for endomorphisms.

Later W. Krieger [67] generalized these results. He proved that every ergodic automorphism $T$ has a generator with $\left[e^{h(T)}\right]+1$ elements.

\subsection{Automorphisms of compact groups}

If an endomorphism of a compact group is ergodic does it have completely positive entropy? 
Rokhlin [37] proved that this is true for commutative groups. Yuzvinsky [55] generalized the result to arbitrary compact groups. Several papers $[3,7,42,57]$ are devoted to the calculation of the entropy of a group endomorphism. The hardest technical problem was solved by Arov in [7]. The most general result is contained in Yuzvinsky's paper [57].

\subsection{Entropy of a skew product}

If $U$ is an automorphism of a space $X$ and $\left\{S_{x}\right\}_{x \in X}$ is a measurable family of automorphisms of a space $Y$ then the formula $T(x, y)=\left(U x, S_{x} y\right)$ defines an automorphism of $X \times Y$. This automorphism $T$ is said to be the skew product with the base $U$ and the fibers $S_{x}$. The problem is to express $h(T)$ in terms of some characteristics of $U$ and $S_{x}$.

This problem was solved by Abramov and Rokhlin in [5] and by R. Adler in [6]. If $\xi$ is a partition of $Y$ with finite entropy and $x \in X$ then we let

$$
\begin{gathered}
\xi_{x}^{n}=\bigvee_{k=0}^{n-1} S_{x}^{-1} S_{U x}^{-1} \cdots S_{U^{k-1} x}^{-1} \xi, \\
h_{U}(S, \xi)=\lim _{n \rightarrow \infty} \frac{1}{n} \int_{X} H\left(\xi_{x}^{n}\right) d x,
\end{gathered}
$$

and

$$
h_{U}(S)=\sup _{\xi} h_{U}(S, \xi)
$$

As it is shown in [5], $h(T)=h(U)+h_{U}(S)$. If in particular $U$ is the identity then $h(T)=\int_{X} h\left(S_{x}\right) d x$. This formula was proved earlier by Rokhlin in [31] and by $\mathrm{K}$. Jacobs in [15].

\section{ET, Leningrad seminar}

In 1960, A. Alexandrov, at that time the Rector of the Leningrad University, offered Rokhlin a position in the Department of Mathematics and Mechanics. It was the beginning of a new period for both Rokhlin and the Department. Being the second best Department of Mathematics in the country, it had had no specialists in ergodic theory and topology prior to 1960 . The ergodic theory seminar, which Rokhlin immediately organized, attracted several young mathematicians already well-known in other areas of mathematics. This group included I. Ibragimov (probability theory), A. Kagan (statistics), B. Makarov, V. Sudakov, A. Vershik (all functional analysis), and L. Abramov who followed Rokhlin to Leningrad and started to work in mathematical economics. Although most of the participants of the seminar did not work in ergodic theory, Rokhlin's influence is clearly noticeable in a number of their papers such as $[16,17,21,48]$. For A. Vershik, ergodic theory became one of the (many) areas of interest for a long time. He developed an elegant theory of Gaussian systems $[49,50]$, solved the long standing problem about a measurable realization of a continuous flow [51], and started the theory of sequences of measurable partitions and their non-measurable limits $[\mathbf{5 2}, \mathbf{5 3}]$. 
The seminar was also a good school for University students. Two of them wrote their PhD theses under Rokhlin's supervision and continued to work in the seminar. R. Belinskaya proved the existence of a derived automorphism with a quasi-discrete spectrum [8] and then followed Vershik in the study of the partition into orbits defined by an automorphism [9]. Yuzvinsky studied endomorphisms on groups and homogeneous spaces $[54,55,57-59]$. Besides the above mentioned results on endomorphisms of compact groups he also considered Halmos' problem about transformations of locally compact but non-compact groups. Can a (Haar) measurepreserving automorphism of such a group be ergodic [13]? It was proved in $[54,58]$ and also in [64-66] that the answer is negative if the group is either commutative or connected. The negative result in the general case was obtained only recently by N. Aoki [61]. Yuzvinsky also proved that automorphisms with a simple spectrum form an everywhere dense $G_{\delta}$ in the space of all automorphisms [56] and followed Vershik in the study of sequences of partitions [60].

Rokhlin himself continued to work in ergodic theory from 1960 to 1967 . Besides the already mentioned results he has also provided an axiomatic definition of entropy [35]. His lecture course [39] on entropy theory is still the best text for a first reading on the subject. This lecture course was the last of Rokhin's publications on ergodic theory. He kept encouraging others to work in the area, attended the seminar, and tried to understand the new methods discovered by Ornstein, but his active research became completely devoted to the topology of 4-dimensional manifolds and real algebraic curves.

\section{B IBLIOGRAPHY}

[1] L. Abramov. The entropy of derived automorphism. Dokl. Akad. Nauk SSSR 128 (1959), 647-650.

[2] L. Abramov. On the entropy of a flow. Dokl. Akad. Nauk SSSR 128 (1959), 873-876.

[3] L. Abramov. The entropy of an automorphism of a solenoidal group. Teor. Veroyatnost. i ee Primen. 4 (1959), 249-254.

[4] L. Abramov. Metric automorphisms with quasi-discrete spectrum. Izv. Akad. Nauk SSSR ser. mat. 26 (1962), 513-530.

[5] L. Abramov \& V. Rokhlin. Entropy of a skew product of mappings with invariant measure. Vestnik Leningrad. Univ. 17 (7) (1962), 5-13.

[6] R. Adler. A note on the entropy of skew product transformations. Proc. Amer. Math. Soc. 14 (1963), 665-669.

[7] D. Arov. The calculation of entropy for a class of group endomorphisms. Zap. Meh. Mat. Fak. $i$ Kharkov. Mat. Obshch. 30 (1964), 48-69.

[8] R. Belinskaya. Existence of a derived automorphism with quasi-discrete spectrum. Mat. Zametki 3 (1968), 539-540.

[9] R. Belinskaya. Partitions of Lebesgue space into orbits defined by ergodic automorphisms. Funkt. Analiz i ego Priloz. 2 (3) (1968), 4-16.

[10] P. Halmos \& J. von Neumann. Operator methods in classical mechanics 2. Ann. of Math. 43 (1942), 332-350.

[11] P. Halmos. On automorphisms of compact groups. Bull. Amer. Math. Soc. 49 (1943), 619-624.

[12] P. Halmos. In general a measure preserving transformation is mixing. Ann. of Math. 45 (1944), 786-792.

[13] P. Halmos. Lectures on ergodic theory. Tokyo, 1956.

[14] E. Hopf. Ergodentheorie, Ergenbnisse der Mathematik, Bd. 5. Springer: Berlin, 1937.

[15] K. Jacobs. Ergodic decomposition of the Kolmogorov-Sinai invariant. in: Ergodic Theory, pp. 173-190. Academic Press: New York, 1963. 
[16] I. Ibragimov. A theorem from the metric theory of continuous fractions. Vestnik Leningrad. Univ. I (1961), 13-24.

[17] I. Ibragimov. The spectrum of stationary Gaussian sequences satisfying the strong mixing condition, 1. Necessary conditions. Teor. Veroyatnost. i ee Primen. 10 (1965), 95-116.

[18] A. Katok \& A. Stepin. Approximations in ergodic theory. Uspekhi Mat. Nauk 22 (5) (1967), 81-106.

[19] A. Kolmogorov. New metric invariants of transitive dynamical systems and automorphisms of a Lebesgue space. Dokl. Akad. Nauk SSSR 119 (1958), 861-864.

[20] A. Kolmogorov. The entropy in a unit of time as a metric invariant of automorphisms Dokl. Akad. Nauk SSSR 124 (1959), 754-755.

[21] B. Makarov. Examples of a singular measure equivalent to its own square by convolution. Vestn. Leningrad. Univ. 7 (1968), 51-54.

[22] D. Ornstein. Bernoulli shifts with the same entropy are isomorphic. Advances in Math. 4 (1970), 337-352.

[23] D. Ornstein. An example of a Kolmogorov automorphism that is not a Bernoulli shift. Advances in Math. 10 (1973), 49-62.

[24] M. Pinsker. The notion of regularity of random processes. Teoriya Veroyatnost. i ee Primen. 4 (1959) 475-476.

[25] V. Rokhlin. On a classification of measurable partitions. Dokl. Akad. Nauk. SSSR 58 (1947), 29-32.

[26] V. Rokhlin. Unitary rings. Dokl. Akad. Nauk SSSR 59 (1948), 643-646.

[27] V. Rokhlin. A 'general' measure-preserving transformation is not mixing. Dokl. Akad. Nauk SSSR 60 (1948), 349-351.

[28] V. Rokhlin. On the fundamental ideas of measure theory. Mat. Sb. 25 (1949), 107-150.

[29] V. Rokhlin. Selected topics from the metric theory of dynamical systems. Uspekhi Mat. Nauk 4 (2) (1949), 57-128.

[30] V. Rokhlin. On endomorphisms of compact commutative groups. Izv. Akad. Nauk SSSR, ser. mat. 13 (1949), 329-340.

[31] V. Rokhlin. On the entropy of a metric automorphism. Dokl. Akad. Nauk SSSR 124 (1959), 980-983.

[32] V. Rokhlin. New progress in the theory of transformations with invariant measure. Uspekhi Mat. Nauk 15 (4) (1960), 3-26.

[33] V. Rokhlin. Exact endomorphisms of a Lebesgue space. Izv. Akad. Nauk SSSR, ser. mat. 25 (1961), 499-530.

[34] V. Rokhlin. The entropy of an automorphism of a compact commutative group. Teoriya Veroyatnost. i ee Primen. o (1961), 351-352.

[35] V. Rokhlin. An axiomatic definition of the entropy of a transformation with invariant measure. Dokl. Akad. Nauk SSSR 148 (1963), 779-781.

[36] V. Rokhlin. Generators in ergodic theory. Vestnik Leningrad. Univ. 1 (1963), 26-32.

[37] V. Rokhlin. Metric properties of endomorphisms of compact commutative groups. Izv. Akad. Nauk SSSR, ser. mat. 28 (1964), 867-874.

[38] V. Rokhlin. Generators in ergodic theory II. Vestnik Leningrad. Univ., 20 (13) (1965), 68-72.

[39] V. Rokhlin. Lectures on the entropy theory of transformations with invariant measure. Uspekhi Mat. Nauk 22 (5) (1967), 3-56.

[40] V. Rokhlin \& S. Fomin. Spectral theory of dynamical systems. in: Proc. Third All-Union Math. Congress, vol. 3, pp. 284-292. Akad. Nauk SSSR: Moscow, 1958.

[41] V. Rokhlin \& Ya. Sinai. Construction and properties of invariant measurable partitions. Dokl. Akad. Nauk SSSR 141 (1961), 1038-1041.

[42] Ya. Sinai. On the notion of entropy of a dynamical system. Dokl. Akad. Nauk SSSR 124 (1959), 768-771.

[43] Ya. Sinai. Dynamical systems and stationary Markov processes. Teoriya Veroyatnost. i ee Primen. 5 (1960), 335-338.

[44] Ya. Sinai. Central limit theorem for geodesic flows on manifolds of constant negative curvature. Dokl. Akad. Nauk SSSR 133 (1960), 1303-1306.

[45] Ya. Sinai. K-systems and geodesic flows on manifolds of constant negative curvature. Dissertation in Phys.-Math. Sci., Moscow State Univ.: Moscow 1960.

[46] Ya. Sinai. Geodesic flows on compact surfaces of negative curvature. Dokl. Akad. Nauk SSSR 136 (1961), 549-552. 
[47] Ya. Sinai. Dynamical systems with countably multiple Lebesgue spectrum 1. Izv. Akad. Nauk SSSR, ser. mat. 25 (1961), 899-924.

[48] V. Sudakov. The existence of variables independent of several given ones. in: Trans. 4th Prague Conf. Information Theory, Statistical Decision Functions. Random Processes, 1965, pp. 589-594. Prague 1967.

[49] A. Vershik. On the theory of normal dynamical systems. Dokl. Akad. Nauk SSSR 144 (1962), 9-12.

[50] A. Vershik. The spectral and metric isomorphisms of certain normal dynamical systems. Dokl. Akad. Nauk SSSR 144 (1962), 255-257.

[51] A. Vershik. Measurable realization of continuous automorphism groups of a unitary ring. Izv. Akad. Nauk SSSR, ser. mat. 29 (1965), 127-136.

[52] A. Vershik. Theorem on lacunary isomorphisms of monotonic sequences of partitions. Funkt. Analiz i ego Priloz. 2 (3) (1968), 17-21.

[53] A. Vershik. Decreasing sequences of measurable partitions and their applications. Dokl. Akad. Nauk SSSR 193 (1970), 748-751.

[54] S. Yuzvinsky. Metric properties of automorphisms of locally compact commutative groups. Sibirsk. Mat. Zh. 6 (1965), 244-267.

[55] S. Yuzvinsky. Metric properties of endomorphisms of compact groups. Izv. Akad. Nauk SSSR, ser. mat. 29 (1965), 1295-1338.

[56] S. Yuzvinsky. Metric automorphisms with a simple spectrum. Dokl. Akad. Nauk SSSR 172 (1967), 1036-1038.

[57] S. Yuzvinsky. Computation of the entropy of a group endomorphism. Sibirsk. Mat. Zh. 8 (1967), 230-239.

[58] S. Yuzvinsky. The absence of ergodic automorphisms on non-compact connected groups. Uch. Zap. Leningrad. Ped. Inst. 387 (1968), 257-262.

[59] S. Yuzvinsky. Metric properties of endomorphisms on homogeneous spaces of compact groups. Izv. Akad. Nauk SSSR, ser. mat. 35 (1971), 78-82.

[60] S. Yuzvinsky. On the entropy of a sequence of partitions generated by an endomorphism. Funkt. Analiz i ego Priloz. 6 (3) (1972), 87-88.

[61] N. Aoki. Dense orbits of automorphisms and compactness of groups. Topology and its Applications 20 (1985), 1-15.

[62] H. Furstenberg. Ergodic behavior of diagonal measures and a theorem on arithmetic progressions. J. d'Analyse Math. 31 (1977), 204-256.

[63] S. A. Kalikow. Twofold mixing implies threefold mixing for rank one transformations. Ergod. Theory \& Dynam. Sys. 4 (1984), 237-259.

[64] R. Kaufman \& M. Rajagopalan. On automorphisms of a locally compact group. Mich. Math J. 13 (1966), 373-374.

[65] M. Rajagopalan. Ergodic properties of automorphisms of a locally compact group. Proc. Amer. Math. Soc. 17 (1966), 372-376.

[66] T. S. Wu. Continuous automorphisms on locally compact groups. Mat, Z. 96 (1967), 256-259.

[67] W. Krieger. On entropy and generators of measure-preserving transformations. Trans. Amer. Math. Soc. 149 (1970), 453-464. 